\title{
Evaluation of the Right Ventricular Function in Hypertrophic Obstructive Cardiomyopathy: A Strain and Tissue Doppler Study
}

\author{
D. ZEMÁNEK ${ }^{1}$, P. TOMAŠOV ${ }^{1}$, P. PŘICHYSTALOVÁ ${ }^{2}, K_{\text {. LINHARTOVÁ }}{ }^{\text {, }}$ \\ J. VESELKA ${ }^{1}$
}

${ }^{1}$ Department of Cardiology, Cardiovascular Center, University Hospital Motol, First and Second Medical School of Charles University, Prague, Czech Republic, ${ }^{2}$ Institute of Mathematics, Faculty of Mechanical Engineering, Brno University of Technology, Brno, Czech Republic

Received August 20, 2009

Accepted February 19, 2010

On-line April 20, 2010

\section{Summary}

We investigated the utility of strain, strain rate, and tissue Doppler imaging (TDI) for the evaluation of the right ventricle (RV) impairment in patients with a hypertrophic obstructive cardiomyopathy (HOCM) who underwent a successful alcohol septal ablation (ASA) and were without RV hypertrophy. A group of 19 patients suffering from HOCM with 22 controls was compared. The parameters of TDI were evaluated in mitral and tricuspid annulus. Strain and strain rate derived from TDI were assessed in an apical free wall of RV, as well as in basal segments of the left ventricle. Between both groups, there were significant differences only in isovolumic pre-ejection time $(79.2 \pm 17.3 \mathrm{~ms}$ vs. $58.5 \pm 8.1 \mathrm{~ms}, \mathrm{p}<0.01)$, isovolumic relaxation time $(104.7 \pm 26.2 \mathrm{~ms}$ vs. $77.3 \pm 24.5 \mathrm{~ms}, \mathrm{p}<0.01)$, myocardial performance (Tei) index measured from TDI ( $0.61 \pm 0.14$ vs. $0.49 \pm 0.09, p<0.01)$, and early peak diastolic velocity of TDI $(10.6 \pm 1.67 \mathrm{~cm} / \mathrm{s}$ vs. $12.6 \pm 2.21 \mathrm{~cm} / \mathrm{s} ; \mathrm{p}<0.05)$. Our results suggest the impairment of both systolic and diastolic RV function in patients suffering from HOCM. TDI-related parameters appear to be more sensitive than strain and strain rate for evaluation.

\section{Key words}

Hypertrophic obstructive cardiomyopathy - Right ventricle • Strain • Strain rate $\bullet$ Tissue Doppler imaging • Tei index

\section{Corresponding author}

David Zemanek, Department of Cardiology, Cardiovascular Center of University Hospital Motol, V Uvalu 84, 15000 Prague, Czech Republic. E-mail: zejada@seznam.cz

\section{Introduction}

Hypertrophic obstructive cardiomyopathy (HOCM) is characterized by myocardial hypertrophy with the impairment of myocardial contraction. Conventional echocardiography provides a useful morphological information for the diagnosis of HOCM, the most characteristic finding among them being the myocardial hypertrophy of the left ventricle (LV) (Elliott et al. 2008). Tissue Doppler imaging (TDI), strain and strain rate (SR) imaging are relatively newly developed echocardiographic techniques that allow a better assessment of regional myocardial function. Several studies have suggested that TDI of mitral annulus, strain, and SR offer more sensible tools for a functional evaluation of the left ventricle in HOCM patients (Nagueh et al. 2003, Kato et al. 2004). The above mentioned techniques provide possibilities to differentiate the patient suffering from HOCM from a healthy subject in the absence of hypertrophy, and to distinguish HOCM between other diseases with hypertrophy such as hypertension and "athletes" heart. TDI of tricuspid annulus was also identified as an independent prognostic factor in patients suffering from HOCM (Efthimiadis $e t$ al. 2007). Since HOCM is a global disease of the myocardium due to genetic defect, we assume that strain, SR, and TDI should be sensitive parameters to find any differences between right ventricle (RV) in patients suffering from HOCM and normal subjects. Up to now, $\mathrm{RV}$ in HOCM has been studied only by TDI, but not examined by strain and strain rate (Mörner et al. 2008). 
The aim of our study was evaluated RV by strain and SR and TDI parameters together.

\section{Methods}

Seventy-five consecutive patients after alcohol septal ablation (ASA) for HOCM with an obstruction underwent simultaneous clinical and echocardiographic investigation between January 2007 and November 2008. Inclusion criteria for involvement in this study were a successful ASA for HOCM with the obstruction more than one year after the treatment. Exclusion criteria were a poor echocardiographic image quality, resting obstruction in the left ventricular outflow tract (LVOT) pressure gradient more than $15 \mathrm{~mm} \mathrm{Hg}$, present hypertrophy of RV (wall thickness $\geq 6 \mathrm{~mm}$ ), echocardiographic markers of pulmonary hypertension (peak systolic gradient on tricuspid valve more than $30 \mathrm{~mm} \mathrm{Hg}$ ), implantation of pacemaker, moderate to severe valve disease, coronary artery disease, pulmonary and liver disease. The group finally consisted of 19 patients. This group was then compared with 22 subjects of control group matched for sex, age and major cardiovascular risk factors (hypertension, diabetes, smoking, and hyperlipidemia).

Echocardiography images were obtained in the parasternal long- and short-axis views, and apical 2- and 4-chamber views, using a standard transducer position with a Philips 7500 ultrasound system (Philips, Andover, MA, USA) by a single operator, and subsequently analyzed by two experienced cardiologists. The parameters of $\mathrm{LV}, \mathrm{RV}$, left and right atrium, and wall thickness, were measured in a parasternal long-axis or apical 4-chamber view. The hypertrophy was calculated according to the recommendation of the American Society of Echocardiography with limitation of asymmetrical distribution of LV hypertrophy (Lang et al. 2005). The volume of LV, left atrium, and ejection fraction of LV were calculated by a simplified Simpson's method in a monoplane projection.

TDI parameters were measured in a septal margin of mitral annulus for LV, and in a lateral margin of tricuspid annulus for RV from pulsed Doppler. Longitudinal strain and SR were obtained from a basal segment of LV (lateral, anterior and inferior wall), and in an apical half of the free wall of RV. The basal segment of interventricular septum was excluded from the analysis, because of being affected by ASA. Apical half of the RV free wall was evaluated thanks to a better image quality. Using the dedicated software (Q-Lab,

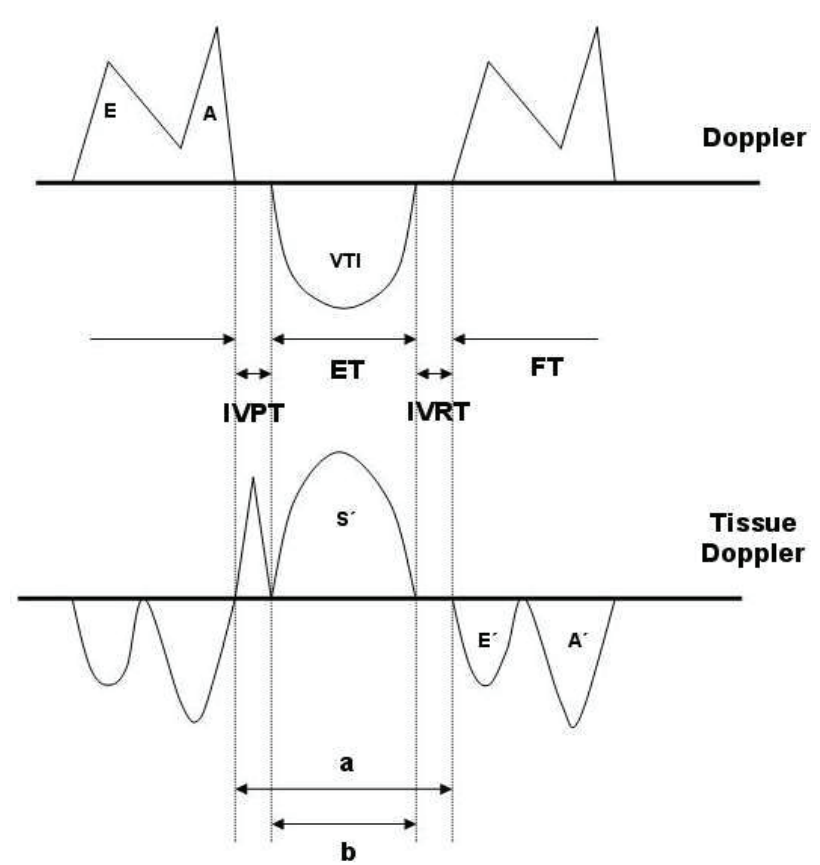

Fig. 1. Schema of the relation between Doppler and Tissue Doppler parameters during the heart cycle (IVPT - isovolumic pre-ejection time, ET - systolic ejection time, IVRT - isovolumic relaxation time, FT - diastolic filling time, a - mitral closing-toopening time, $\mathrm{b}$ - ejection time $=\mathrm{ET}, \mathrm{E}-$ early diastolic peak flow, A - late diastolic peak flow, VTI - velocity time integral in outflow tract of the ventricle, $S^{\prime}-$ peak systolic velocity, $E^{\prime}$ peak early diastolic velocity, $A^{\prime}$ - peak late diastolic velocity)

Philips, The Netherlands), strain and SR were measured from a color Doppler imaging off-line. Strain and SR profiles were averaged over three consecutive cardiac cycles and integrated over time to derive natural strain profiles using an end-diastole as the reference point. Peak systolic SR ("first negative peak" - SRs), peak early diastolic SR ("first positive peak" - SRe), and peak strain (maximal negative deviation - PS) were measured from strain and strain rate (Marwick 2006). TDI was analyzed using the following parameters: peak systolic velocity $\left(S^{\prime}\right)$, peak early diastolic velocity (E'), peak late diastolic velocity $\left(A^{\prime}\right)$, isovolumic pre-ejection time (time interval between the end of the late diastolic wave and the onset of the systolic wave - IVPT), isovolumic relaxation time (time interval between the end of the systolic wave and the onset of the diastolic wave - IVRT), and myocardial performance index (this index is defined as $(a-b) / b$, where $a$ is the interval between the end and the onset of the tricuspid inflow, and $b$ is the ejection time of RV outflow - Tei index) (Fig. 1). Using the TDI for the measurement RV time intervals and Tei index has been previously certified (Harada et al. 2002).

The categorical variables are described with numbers and percentages. All the values were expressed 
Table 1. Clinical characteristics of patients with hypertrophic obstructive cardiomyopathy (HOCM) and controls.

\begin{tabular}{llll}
\hline & HOCM group & Control group & P value \\
\hline Age (years) & $55.2 \pm 10.4$ & $55.6 \pm 7.7$ & n.s. \\
Sex (male, \%) & 42 & 50 & n.s. \\
BSA (m) & $1.9 \pm 0.2$ & $2.0 \pm 0.2$ & n.s. \\
Systolic pressure (mm Hg) & $132.6 \pm 10.7$ & $134.4 \pm 10.3$ & n.s. \\
Diastolic pressure (mm Hg) & $83.2 \pm 9.8$ & $83.9 \pm 8.5$ & n.s. \\
Heart rate (beat/min) & $63.2 \pm 7.2$ & $68.5 \pm 8.3$ & $<0.05$ \\
Hypertension (\%) & 58 & 68 & n.s. \\
Diabetes mellitus (\%) & 11 & 9 & n.s. \\
Dyslipidemia (\%) & 37 & 41 & n.s. \\
Smoking (\%) & 32 & 27 & n.s. \\
Beta-blockers (\%) & 89 & 45 & $<0.05$ \\
Ca channel antagonists (\%) & 18 & n.s. \\
ACE inhibitors (\%) & 11 & 41 & n.s. \\
\hline
\end{tabular}

BSA - body surface area, ACE - angiotensin-converting enzyme

Table 2. Parameters of left ventricle and left atrium in HOCM patients and controls.

\begin{tabular}{llll}
\hline & HOCM group & Control group & P value \\
\hline LVEDD $(\mathrm{mm})$ & $43.4 \pm 3.8$ & $46.9 \pm 3.8$ & $<0.01$ \\
LVESD (mm) & $25.5 \pm 3.0$ & $29.0 \pm 3.5$ & $<0.01$ \\
LV max. thickness $(\mathrm{mm})$ & $18.7 \pm 2.7$ & $10.4 \pm 1.0$ & $<0.01$ \\
EF $(\%)$ & $71.6 \pm 4.9$ & $66.5 \pm 5.1$ & $<0.01$ \\
LVH/BSA $\left(\mathrm{g} / \mathrm{m}^{2}\right)$ & $136.0 \pm 14.7$ & $86.8 \pm 15.8$ & $<0.01$ \\
LA $(\mathrm{ml})$ & $71.4 \pm 16.0$ & $48.9 \pm 11.2$ & $<0.01$ \\
E' (cm/s) & $5.4 \pm 1.7$ & $8.4 \pm 2.3$ & $<0.01$ \\
A' (cm/s) & $7.6 \pm 1.7$ & $9.6 \pm 2.0$ & $<0.01$ \\
LVOT gradient (mm Hg) & $8.5 \pm 3.3$ & $5.2 \pm 1.2$ & $<0.01$ \\
PS septal (\%) & $6.9 \pm 4.5$ & $11.9 \pm 3.8$ & $<0.01$ \\
PS lateral (\%) & $11.3 \pm 2.6$ & $16.2 \pm 2.6$ & $<0.01$ \\
PS inferior (\%) & $8.7 \pm 2.2$ & $12.6 \pm 2.8$ & $<0.01$ \\
PS anterior (\%) & $11.9 \pm 3.9$ & $16.8 \pm 3.6$ & $<0.01$ \\
\hline
\end{tabular}

LVEDD - LV end-diastolic diameter, LVESD - LV end-systolic diameter, EF - ejection fraction, LVH/BSA - left ventricular hypertrophy/body surface area, LA - left atrium, E' - TDI peak early diastolic velocity, A' - TDI peak late diastolic velocity, LVOT - the left ventricular outflow tract, PS - peak strain.

as mean \pm S.D. Data were compared with unpaired T-test to estimate the difference between the two selected groups. The normal (Gaussian) probability distribution was confirmed for this testing. The differences were considered significant at $\mathrm{P}<0.05$.

\section{Results}

Clinical and demographic characteristics of both groups are presented in Table 1. The groups were not significantly different in sex, age, body surface area, blood pressure, and in the presence of main cardiovascular risk factors. We only found a difference in heart rate which resulted from a high prevalence of using beta-blockers in HOCM group. There was no difference in other cardiovascular medications, such as ACE inhibitors, $\mathrm{AT}_{1}$ blockers and calcium channel antagonists. The echocardiographic parameters of LV and left atrium 
Table 3. Parameters of right ventricle and right atrium in HOCM patients and controls.

\begin{tabular}{llll}
\hline & HOCM group & Control group & P value \\
\hline Diameter of $R V(\mathrm{~mm})$ & $24.9 \pm 2.8$ & $23.5 \pm 1.9$ & n.s. \\
Diameter of $R A(\mathrm{~mm})$ & $50.2 \pm 4.7$ & $49.5 \pm 4.2$ & n.s. \\
TAPSE $(\mathrm{mm})$ & $2.9 \pm 0.3$ & $2.9 \pm 0.3$ & n.s. \\
ACT $(\mathrm{ms})$ & $136.8 \pm 16.6$ & $121.1 \pm 32.5$ & n.s. \\
$S^{\prime}(\mathrm{cm} / \mathrm{s})$ & $15.4 \pm 4.7$ & $15.8 \pm 2.8$ & n.s. \\
$E^{\prime}(\mathrm{cm} / \mathrm{s})$ & $11.2 \pm 1.8$ & $12.9 \pm 2.6$ & $<0.05$ \\
$A^{\prime}(\mathrm{cm} / \mathrm{s})$ & $16.8 \pm 4.1$ & $17.6 \pm 4.0$ & n.s. \\
IVPT $(\mathrm{ms})$ & $79.2 \pm 17.3$ & $58.5 \pm 8.1$ & $<0.01$ \\
$I V R T(\mathrm{~ms})$ & $104.7 \pm 26.2$ & $77.3 \pm 24.5$ & $<0.01$ \\
Tei $\mathrm{index}$ & $0.61 \pm 0.14$ & $0.49 \pm 0.09$ & $<0.01$ \\
PS $(\%)$ & $17.7 \pm 5.6$ & $21.6 \pm 9.1$ & n.s. \\
SRs $\left(\mathrm{s}^{-1}\right)$ & $1.2 \pm 0.3$ & $1.2 \pm 0.3$ & n.s. \\
SRe $\left(\mathrm{s}^{-1}\right)$ & $1.2 \pm 0.4$ & $1.3 \pm 0.5$ & n.s. \\
\hline
\end{tabular}

RV - right ventricle, RA - right atrium, TAPSE - tricuspid annular plane systolic excursion, ACT - acceleration time, $S^{\prime}-$ TDI peak systolic velocity, E' - TDI peak early diastolic velocity, $\mathrm{S}^{\prime}$ - TDI peak late diastolic velocity, IVPT - isovolumic pre-ejection time, IVRT isovolumic relaxation time, Tei index - myocardial performance index, PS - peak strain (maximal negative deviation), SRs - peak systolic strain rate, $\mathrm{SRe}$ - peak early diastolic strain rate.

are summarized in Table 2. These results are in agreement with the previous evidence of impaired systolic and diastolic LV functions in HOCM. We confirmed significant difference for strain (peak strain) for basal segments of LV (lateral, inferior and anterior) between HOCM and controls. Septal basal segment was excluded due to a possible affection by the scar after ASA. The parameters of $\mathrm{RV}$ and right atrium are presented in Table 3. There was no difference in conventional parameters of RV either. Among the parameters of tricuspid annulus TDI, we observed a significant difference in the value of early diastolic wave (E') and time intervals measured from TDI (IVPT, IVRT, and RV myocardial performance (Tei) index). We found no significant difference for strain and SR, only a trend for PS of RV was observed.

The reproducibility of technical and logical strain and strain rate measurement was confirmed by intra- and interobserver variability, which is also agreed with previous references and has a similar value for RV and LV (strain: intraobserver $8.8 \%$ and interobserver variability $12.6 \%$, SR: intraobserver $16.6 \%$ and interobserver variability $18 \%$ ).

\section{Discussion}

We demonstrated a functional impairment of RV in HOCM patients without a significant RV hypertrophy in our study. Moreover, to the best of our knowledge, this study is the first one to demonstrate the higher sensitivity of TDI compared to strain and SR derived from TDI in the evaluation of RV function.

The IVPT represents a systolic function, the IVRT represents a diastolic function and the Tei index is more complex. We found no significant difference for strain and SR in measured parameters. Despite the above mentioned findings, we confirmed the difference for strain and strain rate in LV. These results suggest that the impairment of non-hypertrophied RV in contrast to the left ventricle appears to be less pronounced. The TDI of tricuspid annulus seems to be more sensitive to detect these mild abnormalities than strain and strain rate.

The changes of the left ventricle in HOCM are well recognized and described, but in the case of the right ventricle the situation is quite different. The problem was in evaluation of RV that is influenced by dominant LV and that is also highly load-sensitive (Mandysová and Niederle 2007). The morphological impairment of RV in HOCM was recently documented by a magnetic resonance (Maron et al. 2007). Our findings confirmed the previously reported systolic and diastolic impairment of RV measured by TDI similar to previous study (Mörner et al. 2008) also for non-hypertrophied RV. The $\mathrm{RV}$ dysfunction is suggested mainly a primary 
phenomenon related directly to a genetic defect of RV myocardium.

However, IVPT and IVRT are affected by a number of hemodynamic and electromechanical variables, including heart rate. Hence Tei et al. (1995) proposed a "myocardial performance index" (or "Tei index") that is independent of the electromechanical delay. The Tei index has been found to be of a prognostic and clinical value in a number of heart disease including HOCM (Mikkelsen et al. 2006, Veselka et al. 2005). Although the Tei index has been proposed for the evaluation of the LV function, it can also be useful for the RV investigation (Vonk et al. 2007). The use of TDI for the measurement RV time intervals has been previously validated (Harada et al. 2002). In our study we observed a significant difference for the Tei index, corroborating the prolongation of IVPT and IVRT, confirming the impairment of both systolic and diastolic RV functions.

The failure of strain and strain rate derived from TDI to detect abnormalities in systolic function may be explained by several limitation of this technique including angle dependence, apico-basal gradient, etc. (Marwick 2006). The apico-basal gradient for strain and strain rate parameters was observed for $\mathrm{LV}$ in previous studies. LV basal segments have a better diagnostic value than the apical segments for the determination of HOCM (Serri et al. 2006). However, we evaluated only an apical half of RV free wall since it was impossible to obtain a good quality image of the basal part of non-hypertrophied $\mathrm{RV}$ in most of our patients, especially upward tricuspid annulus. Another possible reason could be a limitation caused by an angle deviation between the RV free wall longitudinal axis and the axis of measurement in some patients from an apical view. In addition, the TDI of the tricuspid annulus evaluates global changes in RV, while strain and SR the regional ones. Although a similar study with hypertensive patients showed a significant difference for the RV peak systolic strain $(p<0.05)$, the TDI of tricuspid annulus was more sensitive than strain and SR in this study as well (Tumuklu et al. 2007). The prevalence of hypertension was similar between groups in our study.

The limitation of this study was also a relatively small number of patients. However, HOCM is a relatively rare disease and previous investigations were performed with a similar number of patients (Nagueh et al. 2003, Kato et al. 2004, Serri et al. 2006). Moreover, we evaluated only patients following a successful alcohol septal ablation, who represent a small subgroup within the total HOCM cohort. We studied these patients because they represent a relatively homogenous group, and because the ASA excluded the hemodynamic influence of obstruction in LVOT to left ventricle and subsequently right ventricle function. The function of RV free wall should not be influenced by the interventricular scar after ASA. Our intra- and interobserver variability was in accordance with previous references.

Our findings support a general involvement of the whole myocardium in HOCM due to a genetic defect. Probably due to a technical imaging limitation, the tricuspid annular tissue Doppler imaging seems to be more sensitive to detect an early systolic and diastolic dysfunction of non-hypertrophied RV than strain and strain rate.

\section{Conflict of Interest}

There is no conflict of interest.

\section{Acknowledgements}

This study was supported by grant NR/9164-3 from the Internal Grant Agency, Ministry of Health, Czech Republic and by grant Nr. 000064203 from Ministry of Health, Czech Republic.

\section{References}

EFTHIAMIADIS GK, GIANNAKOULAS G, PARCHARIDOU DG, KARVOUNIS HI, MOCHSLAS ST, STYLIADIS IH, PAPADOPOULOS CE, KOUNATIADIS P, PLIAKOS CI, PARCHARIDIS GE, LOURIDAS GE: Clinical significance of tissue Doppler imaging in patients with hypertrophic cardiomyopathy. Circ J 71: 897-903, 2007.

ELLIOTT P, ANDERSSON B, ARBUSTINI E, BILINSKA Z, CECCHI F, CHARRON P, DUBOURG O, KÜHL U, MAISCH B, MCKENNA WJ, MONSERRAT L, PANKUWEIT S, RAPEZZI C, SEFEROVIC P, TAVAZZI L, KEREN A: Classification of the cardiomyopathies: a position statement from the European Society Of Cardiology Working Group on Myocardial and Pericardial Diseases. Eur Heart $J$ 29: 270-276, 2008. 
HARADA K, TAMURA M, TOYONO M, YASUOKA K: Comparison of the right ventricular Tei index by tissue Doppler imaging to that obtained by pulsed Doppler in children without heart disease. Am J Cardiol 90: 566569, 2002.

KATO TS, NODA A, IZAWA H, YAMADA A, OBATA K, NAGATA K, IWASE M, MUROHARA T, YOKOTA M: Discrimination of nonobstructive hypertrophic cardiomyopathy from hypertensive left ventricular hypertrophy on the basis of strain rate imaging by tissue Doppler ultrasonography. Circulation 110: 3808-3814, 2004.

LANG RM, BIERIG M, DEVEREUX RB, FLACHSKAMPF FA, FOSTER E, PELLIKKA PA, PICARD MH, ROMAN MJ, SEWARD J, SHANEWISE JS, SOLOMON SD, SPENCER KT, SUTTON MS, STEWART WJ: Recommendations for chamber quantification: a report from the American Society of Echocardiography's Guidelines and Standards Committee and the Chamber Quantification Writing Group, developed in conjunction with the European Association of Echocardiography, a branch of the European Society of Cardiology. J Am Soc Echocardiogr 18: 1440-1463, 2005.

MANDYSOVÁ E, NIEDERLE P: Influence of load changes on tricuspid inflow. Physiol Res 56: 299-305, 2007.

MARON MS, HAUSER TH, DUBROW E, HORST TA, KISSINGER KV, UDELSON JE, MANNING WJ: Right ventricular involvement in hypertrophic cardiomyopathy. Am J Cardiol 100: 1293-1298, 2007.

MARWICK TH: Measurement of strain and strain rate by echocardiography: ready for prime time? J Am Coll Cardiol 47: 1313-1327, 2006.

MIKKELSEN KV, MØLLER JE, BIE P, RYDE H, VIDEBAEK L, HAGHFELT T: Tei index and neurohormonal activation in patients with incident heart failure: serial changes and prognostic value. Eur J Heart Fail 8: 599$608,2006$.

MÖRNER S, LINDQVIST P, WALDENSTRÖM A, KAZZAM E: Right ventricular dysfunction in hypertrophic cardiomyopathy as evidenced by the myocardial performance index. Int J Cardiol 124: 57-63, 2008.

NAGUEH SF, MCFALLS J, MEYER D, HILL R, ZOGHBI WA, TAM JW, QUIÑONES MA, ROBERTS R, MARIAN AJ: Tissue Doppler imaging predicts the development of hypertrophic cardiomyopathy in subjects with subclinical disease. Circulation 108: 395-398, 2003.

SERRI K, REANT P, LAFITTE M, BERHOUET M, LE BOUFFOS V, ROUDAUT R, LAFITTE S: Global and regional myocardial function quantification by two-dimensional strain: application in hypertrophic cardiomyopathy. J Am Coll Cardiol 47: 1175-1181, 2006.

TEI C, LING LH, HODGE DO, BAILEY KR, OH JK, RODEHEFFER RJ, TAJIK AJ, SEWARD JB: New index of combined systolic and diastolic myocardial performance: a simple and reproducible measure of cardiac function-a study in normals and dilated cardiomyopathy. J Cardiol 26: 357-366, 1995.

TUMUKLU MM, ERKORKMAZ U, OCAL A: The impact of hypertension and hypertension-related left ventricle hypertrophy on right ventricle function. Echocardiography 24: 374-384, 2007.

VESELKA J, DUCHOŇOVÁ R, PROCHÁZKOVÁ S, PÁLENÍČKOVÁ J, SORAJJA P, TESAŘ D: Effects of alcohol septal ablation for hypertrophic obstructive cardiomyopathy on Doppler Tei index: a midterm follow-up. Echocardiography 22: 105-109, 2005.

VONK MC, SANDER MH, VAN DEN HOOGEN FH, VAN RIEL PL, VERHEUGT FW, VAN DIJK AP: Right ventricle Tei-index: a tool to increase the accuracy of non-invasive detection of pulmonary arterial hypertension in connective tissue diseases. Eur J Echocardiogr 8: 317-321, 2007. 